\title{
Identidad de género: ¿obstáculo al desarrollo o acceso a la equidad?
}

\author{
Ximena Natalia Ortega Delgado \\ Ángela Cristina Delgado Zambrano
}

\begin{abstract}
Abstact
Part of a research proyect carried out among teenagers in the town of Catambuco (Nariño, Colombia), this article exposes and reflects about social representations related to gender and identity that prevail in the attitudes and beliefs of this poblational segment.Here, the authors recognize the existence of stereotypes, opinions, values and norms that form the meaning of being in the world as a man or a woman, and discuss the implications that such representations have on gender equity.
\end{abstract}

\section{Introducción}

Los estudios de género inicialmente fueron una forma de acercarse a las problemáticas de las mujeres, quienes eran las víctimas de una sociedad que promovía insistentemente un sistema patriarcal, en el que por regla el dominio de lo masculino estaba sobre lo femenino, y no se le asignaba a la mujer otras funciones aparte del papel de madre que le otorgaba su propia biología. A lo largo del tiempo la cultura ha continuado promoviendo diversas formas de subvaloración hacia lo considerado femenino y éstas han sido aceptadas por los individuos, quienes generación tras generación heredan a las culturas todos estos legados de desigualdad. Los proyectos que han sido parte de estos estudios y que han sido dirigidos a hombres, a mujeres o a ambos, pueden modificar las condiciones que crean relaciones de desventaja entre los géneros, y ocuparse particularmente de las posiciones de la mujer para lograr una mayor equidad que la vigente. La lucha por la igualdad debe entonces ser comprendida no sólo como un asunto de mujeres, sino como un paso necesario de la humanidad para progresar, entendiendo que el estudio de estos fenómenos debe darse en espacios en los que tanto hombres como mujeres sean partícipes, porque es en 
la interrelación de ambos géneros donde se comprende tal situación. Es por esto que en esta investigación se ha pretendido fortalecer a las representaciones sociales que giran en torno a la identidad de género de los y las adolescentes de Catambuco, desde la implementación de estrategias que fomenten espacios de equidad de género.

Flores subraya que la actitud de separar tan radicalmente a los individuos de acuerdo a su sexo se debe a las desigualdades existentes en la sociedad y al valor que se asigna a las tareas, funciones y roles realizados por hombres o por mujeres. En este proceso es donde la ideología juega un papel importante al desatar las diferencias socialmente establecidas en términos de la oposición natural entre los sexos mientras reduce o elimina las similitudes. ${ }^{1}$ Con esta investigación se ha buscado movilizar los conceptos de identidad femenina y masculina para llegar a una equidad de género en donde los y las adolescentes gocen de espacios donde se favorezcan formas más sanas de interrelación, que promuevan cambios socioculturales a favor de un ambiente saludable que permita el libre desarrollo de hombres y mujeres.

Son muchas las personas y organizaciones que a nivel mundial se han encargado de ofrecerle un nuevo y mejor lugar a las mujeres dentro de la esfera social y de luchar por los derechos de ellas. Los estudios investigativos que han surgido en torno a estos temas consideran necesario centrar la atención en las mujeres, no como una categoría de estudio, sino como un proceso que permite un acercamiento a los fenómenos de discriminación de un grupo conformado por más del $50 \%$ de la población mundial, teniendo en cuenta que el surgimiento de esta discriminación no es algo determinado por la naturaleza. Por ello es necesario desnaturalizarla, devolverla al plano de lo social, donde se genera y puede ser explicada, teniendo la posibilidad de cuestionar las formas elementales de la desigualdad humana. ${ }^{2}$

La Organización Mundial de la Salud (OMS) se encarga de desempeñar las funciones de liderazgo en este tema a nivel mundial, y de realizar investigaciones, normas, artículos, opciones de política basada en la evidencia, prestar apoyo técnico a los países y vigilar las tendencias sanitarias mundiales. La OMS presentó la "Política de Igualdad de Género" en el año 2002, donde contempla trabajar con los gobiernos y la sociedad civil en los Estados miembros y con actores relevantes para eliminar las desigualdades entre hombres y mujeres y avanzar hacia el logro de la igualdad de género y empoderamiento de las mujeres. La

1 Flores P., Fátima, Psicología social y género: El sexo como objeto de representación social, México, McGraw Hill, 2000.

2 Grassi, Estela (comp.), Simposio “Antropología de la mujer"', Congreso Argentino de Antropología Social (agosto de 1986: Argentina), Editorial Humanitas, Argentina, pp. 21 a 36. 
OMS también está comprometida en impulsar la igualdad de género en su propia fuerza laboral.

En Pasto, desde la Alcaldía Municipal, la Oficina de Género y Derechos Humanos aborda temas relacionados con la discriminación por razón de género. Mediante el "Diagnóstico integral sobre la posición y condición de género en el municipio de Pasto", realizado en el ańo 2005 abarcando cinco factores (cultural, social, económico, educativo y político), se pudo determinar que la cultura de subordinación hacia la mujer continua arraigada en nuestros pobladores/as y se determinaron las diferentes formas de vulneración de los derechos de las mujeres. ${ }^{3}$ De esta forma se ha intervenido en el desarrollo de programas para sensibilizar a las personas con el tema de la discriminación de género e implementar proyectos que conlleven a la igualdad en las diferentes esferas sociales.

Asumir el reto de promover y fortalecer el potencial de los y las adolescentes del corregimiento de Catambuco implica la promoción de construcciones sanas de convivencia entre ambos sexos, en donde no se pretenda totalizar a las personas haciéndolas ver a todas como frutos de un mismo árbol, sino considerar que esas diferencias pueden servir para construir una sociedad basada en una verdadera equidad de género donde los ambientes públicos y privados cuenten con la participación de ambos personajes, hombres y mujeres, como protagonistas de cambios sociales en beneficio de la equidad.

\section{Metodología}

Para la presente investigación se utilizó el enfoque crítico-social, con el fin de poder abordar la problemática desde una toma de control de las propias vidas de los y las adolescentes, ya sea de manera personal y/o colectiva. Este enfoque tiene un claro interés que busca la liberación, por lo que también se lo conoce como emancipatorio, término que resalta la actitud develadora y de ruptura de lazos ideológicos que no permiten el libre desenvolvimiento de la conciencia humana, debido al carácter ideológico de los mismos. Con esta investigación se movilizaron los conceptos de identidad femenina y masculina para plantear una equidad de género en donde los y las adolescentes puedan gozar de espacios donde se favorezcan formas más sanas de interrelación y se promuevan cambios socioculturales a favor de un ambiente saludable que permita el libre desarrollo de hombres y mujeres. El trabajo investigativo se enmarcó en la línea de investigación acción (IA), en la cual se realiza una serie de procesos y diferentes actividades que relacionan la práctica con la elaboración teórica, para

3 “Diagnóstico integral sobre la posición y condición de género en el municipio de Pasto”, Alcaldía Municipal de Pasto, 2005. 
así tratar de identificar los problemas, reprogramando y poniendo en movimiento estas dificultades. La investigación acción se centra en la posibilidad de aplicar categorías científicas para la comprensión y mejoramiento de las personas. Por medio de esta investigación se exploraron intereses, necesidades y problemáticas de los y las adolescentes que hicieron parte del grupo que se conformó con fines investigativos y que se denominó "Jóvenes Construyendo Identidad" (JCI).

\section{Resultados y análisis}

Los adolescentes separan lo que significa ser hombre y ser mujer teniendo en cuenta las características de cada género, ya que tienen muy claro el papel que cada uno desarrolla en su comunidad. Siempre los hombres se muestran como la cabeza de la familia, en cambio la mujer debe mantener su papel dedicándose a las labores del hogar, el cuidado de los hijos, el arreglo y aseo de la casa. En este sentido, Martha Lamas refiere "la imponente visión dualista que asimila de manera lineal lo masculino a la esfera pública, la autoridad sobre la familia y el bien común y lo femenino a lo doméstico y los intereses privados, articulando estas oposiciones alrededor de la identificación de la honra del grupo familiar con la pureza sexual femenina". ${ }^{4}$

Según la división de las actividades que deben desempeñar tanto hombres como mujeres, Simone de Beauvoir argumenta:

La reproducción biológica es identificada como el fenómeno de sujeción al cual las mujeres han anclado su historia de tal forma que el rol femenino fue determinado por la función reproductiva, ocasionando la exclusión de las mujeres de diferentes actividades que progresivamente se convirtieron en las más valoradas socialmente, así la división del trabajo en función del sexo significó la variación de la diferencia de las tareas, que se convierten gradualmente en inequidad entre ellos. ${ }^{5}$

De esta manera se puede ver que la idea que resalta Beauvoir hace referencia a la relación que se ha generado entre el papel de las mujeres o rol femenino y la función de reproducción biológicamente asignada a ellas, ocasionando de esta manera que las funciones que hacen referencia al cuidado de los hijos y el hogar sean asumidas como algo natural a las mujeres, mientras que a los hombres se les asigna funciones relacionadas con los ambientes públicos y sociales.

4 Lamas, Martha, "Cuerpo e identidad”, en: Arango, León y Viveros (compiladoras), Género e identidad. Ensayos sobre lo femenino y lo masculino, Tercer Mundo Editores-Ediciones Uniandes, Bogotá, 1995.

5 De Beauvoir, Simone, El segundo sexo, Editorial Cátedra, Madrid, (1949) 1998. 
Los adolescentes se perciben a sí mismos como sujetos similares a los demás hombres de su comunidad, destacando que deben ser ellos los que tienen que "mandar" en la casa, haciendo alusión a que son la autoridad dentro del hogar. Por lo tanto se sienten con el derecho a usar la fuerza física, incluso para "expresarle su amor" a la pareja y se ven en la obligación de llevar las riendas del hogar. En cuanto al tema del maltrato, ellos manifiestan que las mujeres terminan acostumbrándose al trato que ellos les dan, conviertiéndose esta en una justificación frente al tema de la violencia intrafamiliar. Por otra parte, las mujeres aceptan las normas que intrínsecamente se han generado dentro de esta comunidad, se limitan a velar por el cuidado de sus hijos e hijas y del hogar. Dentro de las características que hacen parte de las mujeres de Catambuco, se resalta que para ellas es importante cuidar su reputación y no permitir el "qué dirán" de los demás. En cuanto a este último aspecto mencionado, Martha Lamas refiere en su ensayo "Cuerpo e identidad" que "lo femenino se identifica con la honra del grupo sexual y con la pureza sexual femenina". ${ }^{6}$

Podemos evidenciar cómo dentro del grupo de jóvenes se comparten ideas en común con relación a lo que se cree que le corresponde hacer y ser a cada género. Incluso cuando se habla de la familia y las restricciones que existen para hombres y mujeres podemos contemplar esa presencia de lo que Best y Williams señalan como "reglas" que, aun sin ser expresadas formalmente, conforman lo que se entiende como el "sistema de género" en donde se encuentra el ideal que tiene cada sociedad de sus hombres y mujeres. Con respecto a la situación de las mujeres en Colombia, Useche menciona:

En Colombia el machismo con su odiosa discriminación hacia la mujer todavía está presente en la vida familiar, laboral y social. Son muy pocos los hombres, aun en los grupos socioeconómicos más pobres, que colaboran regularmente en las labores domésticas o en el cuidado de los nińos. Políticamente, la mujer en Colombia alcanzó la igualdad ante la ley apenas en la década del cincuenta pero continúan siendo una minoría en los puestos de representación y en los cargos gubernamentales. ${ }^{7}$

Muchas veces los hombres, por las ideas que se han promovido bajo el manto del machismo y el orgullo propio, se sienten incapacitados para expresar sus sentimientos de una manera adecuada; por lo contrario prefieren ocultarlos, porque mostrarlos abiertamente podría significar debilidad ante los demás. Las

6 Lamas, Martha, “Cuerpo e identidad”, en: Arango, León y Viveros (compiladoras), Género e identidad. Ensayos sobre lo femenino y lo masculino, Tercer Mundo Editores-Ediciones Uniandes, Bogotá, 1995.

7 Useche, B., "La sexualidad colombiana", en: Basic Sexological Premises. Estudios de Sexología. Manizales: ARS Ediciones, 1999. 
expresiones de agresividad e ira son mas frecuentes en los hombres, encontrando mayor aprobación social cuando se comportan de esa manera. Por otro lado, hombres y mujeres coinciden en que las mujeres tienden a verse afectadas por demasiadas cosas con relación al hombre, que son muy propensas a llorar ante algunas situaciones $\mathrm{y}$, en general, son vistas como más sensibles y afectivas frente a los eventos que suceden en el entorno que las rodea. Dentro de la población de adolescentes de Catambuco, los que se consideran como aspectos más representativos de hombres y mujeres en la comunidad son características tales como la dedicación al trabajo, la responsabilidad en las diversas esferas sociales y familiares y los fuertes lazos que tienen con sus familias de origen.

La familia es una institución que tiene sus propias funciones sociales, como por ejemplo transmitir valores, costumbres y tradiciones; en ella se adquieren y desarrollan actitudes, hábitos de trabajo y juicio moral. Es en ella en donde se enseña la lengua, el modo de vestir, la manera de celebrar los nacimientos, de enterrar a los muertos, la manera de pensar y analizar la historia, los modos de relacionarse con la comunidad y con otras personas o grupos sociales. "La familia resulta ser el principal espacio de construcción de identidad a través del proceso de socialización por ser donde la niña o el niño permanece durante su formación y su proceso de identificación primaria, en la cual interioriza valores, normas, actitudes, lenguaje, comportamientos particulares, manejo del poder, manejo de la autoridad y estilos de relación con las personas de su entorno". ${ }^{8}$

Las características asociadas a cada género, empiezan a constituirse a muy temprana edad, con la socialización de género que empieza poco después del nacimiento, cuando la primera pregunta que hacen las personas a los nuevos padres no es “¿está saludable el bebé?”, sino “¿es niño o es niña?". Incluso la gente se incomoda al comentar sobre la apariencia o actividad del bebé o seleccionar un regalo para él, hasta saber el sexo del niño; la respuesta a la pregunta acerca de si es niña o niño tiene consecuencias sociales inmediatas. Los padres al realizar esta pregunta afirman que el sexo radica gran parte de las diferencias anatómicas y fisiológicas entre los hombres y las mujeres. Por lo tanto, los individuos no nacen hechos psicológicamente como hombres o mujeres, sino que la constitución de una identidad sexual es el resultado de un largo proceso, de una construcción que se va tejiendo en interacción con el medio familiar y social. Aunque los roles de género difieren transculturalmente, la estratificación de género es universal (Marini 1990), y lo que predomina es que todas las sociedades ponen un valor cultural mas alto en los roles masculinos que los femeninos. "Por todas partes, 
en cada cultura conocida, las mujeres son consideradas en algún grado inferior al hombre" señala el antropólogo Sherry Ortner.'

Las sociedades humanas son ese entramado de relaciones, en donde cuyos habitantes y su entorno se interrelacionan constantemente. Este grupo conformado por individuos comparten lazos ideológicos, económicos, políticos, sociales y culturales. Aquí podemos encontrar la presencia de ciertas normas o exigencias acerca de lo que espera la sociedad de los individuos, se puede decir que existen ciertos códigos implícitos acerca de que es correcto para hombres, para mujeres y para ambos como miembros de ella y demás instituciones. Los jóvenes resaltan de unos y otros en su comunidad, rescatando las características que consideran más importantes. Así se piensan ambos géneros como actores importantes, resaltando los papeles que tienen dentro de la familia y de la sociedad. En cuanto al mundo laboral, los hombres se muestran algo incómodos al visualizar a una mujer asumiendo cargos de alto nivel jerárquico. Ellas, a su vez, se sienten competentes, incluso mucho mejor que los hombres para desempeñar dichos cargos.

En las concepciones existentes sobre la identidad femenina y la identidad masculina podemos ver que los jóvenes consideran a la identidad masculina como algo que tiene relación con la fuerza característica de los hombres y la relacionan con la concepción de que los hombres deben ser "machos" para afrontar todas las cosas y situaciones, es decir, deben mostrarse tal y como son sin dar explicación a nuevas situaciones que se les presenten. Se destaca la agresividad como una forma de dominación, haciéndose evidente una relación de poder en las interacciones cotidianas ejercidas por parte del hombre, no sólo sobre la mujer, sino también como medio de dominación sobre otros hombres. Según Jorge Corsi:

Se ha comprobado que los hombres han incorporado en su proceso de socialización de género un conjunto de creencias, valores y actitudes que en su configuración más estereotipada delimitan la denominada "mística masculina": restricción emocional, homofobia, modelos de control, poder y competencia, obsesión por los logros y el éxito. ${ }^{10}$

Dentro de esta mística masculina podemos encontrar que el modelo de masculinidad tradicional en las comunidades suele estar asentado en el "mito del héroe", que pervive entre nosotros como estereotipo promedio. Aunque sea cuestionado, este denominado mito del héroe, según Badinter, "hace alusión a la idea de que un verdadero hombre debe ser fuerte, competitivo, exitoso en el

9 Gelles, Richard y Ann Levine, Sociología con aplicaciones en paises de habla hispana. Editorial McGraw Hill, Sexta Edición. México, 2000.

10 Corsi, Jorge y otros, Violencia masculina en la pareja, Editorial Paidos. Buenos Aires, Argentina, 1999. 
trabajo y con las mujeres, valiente y arriesgado aunque deba pagar el costo de sus excesos, autosuficiente y agresivo". ${ }^{11}$ Cabe entonces preguntarse cuánto de esta mística masculina está en la base de las dificultades que exhiben los hombres en el acercamiento afectivo a sus hijos varones y constituye un obstáculo a lo que entendemos como un buen desempeño de la función paternal. Desde el mito del hombre como héroe se aborda el papel que desempeńa el hombre y lo describe como un ser fuerte; sin ser analizada la otra parte, en donde se describa que también tiene cualidades como ser cariñoso, tierno, entregado al hogar. Por eso es necesario replantear las relaciones de género y liberar no sólo a las mujeres de las cadenas que la atan al hogar, sino también a los hombres del estereotipo exclusivo de la fortaleza, para pasar a tener en cuenta su parte emocional.

Las opiniones de los adolescentes en cuanto a la identidad femenina destacan dos aspectos que marcan la diferencia entre las mujeres y los hombres. El primero de ellos es la parte física, donde se hace referencia a todo aspecto corporal que los hace diferentes a ambos (órganos genitales, glándulas mamarias, barba, caderas y demás características en la estructura corporal). En segundo lugar, los adolescentes mencionan características en cuanto a la manera de ser, entre las que se incluye la influencia de las emociones en su forma de actuar, la sencillez, la sensibilidad frente a los acontecimientos, la delicadeza, la ternura y algo muy típico de ellas, como lo mencionan los adolescentes, la capacidad de intuición. De igual manera, cuando los jóvenes hablaron de los aspectos femeninos, mencionaron aspectos éticos y normas morales que promueve su comunidad, refiriéndose a esas normas intrínsecas de la sociedad que marcan la diferencia y que hacen que una mujer sea reconocida como una "buena mujer", tal como lo expresan las adolescentes, cuando ha logrado incorporar todos esos valores promovidos por las diferentes instituciones sociales presentes en la comunidad, ya sea la familia, la iglesia o la escuela.

$\mathrm{Al}$ hacer alusión a los aspectos que hacen parte de lo femenino como de lo masculino, se pudo evidenciar que los adolescentes se valen de elementos de la naturaleza para simbolizarlo. Por ejemplo, la luna y la tierra son vistos como algo femenino y el fuego y el sol como aspectos que representan lo masculino. También se valen de narraciones mitológicas para describir lo femenino y lo masculino, tales como la teoría de la creación que aparece en el libro Génesis de la Biblia, en donde se hace referencia al papel asignado a la mujer y al hombre con los valores promovidos por las instituciones religiosas. Allí se narra que es el hombre quien debe proteger a la mujer de los peligros y luchar por ella y sus hijos, y la mujer debe dedicarse a su familia brindándole los servicios y cuidados necesarios. Los jóvenes consideraron que los elementos femenino y masculino hacen referencia a 
dos opuestos, por lo tanto las características debían ser asignadas sólo a una de las dos categorías, y encontraron dificultades en las características comunes a ambas. En este punto surgió una discusión en la que se buscaron mayores argumentos para considerar una característica como algo femenino o masculino.

En la teoría se puede evidenciar que "al momento de nacer se despliega la lógica del género en la función de la apariencia externa de los genitales, a la criatura se le habla de una cierta manera, se le trata distinto, se le alimenta diferente y se depositan sobre ella ciertas expectativas y deseos. Así arranca el proceso de atribución de características "femeninas" y "masculinas" a cada sexo, a sus actividades y conductas, y a las esferas de su vida." ${ }^{12}$ Así mismo, cabe mencionar a los autores Caicedo, Gómez, Bernal, y García, quienes explican "cómo la identidad de género es el reconocimiento que una persona hace acerca de su yo o de su "sí mismo" como hombre o como mujer, reconocimiento basado en la interacción de las características, valores y creencias que una cultura específica ha establecido como apropiadas para uno u otro sexo y la significación personal que cada hombre o mujer elaboran sobre ellas, a partir de su propia historia de vida". ${ }^{13}$

Aludiendo a las particularidades de esta investigación, se puede resaltar que la población del Corregimiento de Catambuco se caracteriza por sus tradiciones, costumbres y religiosidad, su gente ha evolucionado por los cambios sucedidos, entre ellos la cercanía y los medios de transporte a la ciudad. ${ }^{14} \mathrm{El}$ sector económico se caracteriza por trabajos agrícolas y por ser un centro turístico que atrae con la variedad de restaurantes familiares que se destacan por los platos típicos de la región, entre ellos el cuy. La participación de los habitantes en procesos de transformación social es muy baja, adoptando una postura pasiva frente a los problemas que enfrenta la sociedad. La institución religiosa tiene una alta influencia en la población, ya que es esta la encargada de desarrollar proyectos y atención a la comunidad con las diferentes problemáticas que se presentan. Por ello sus habitantes se encuentran muy aferrados a las normas y valores promovidos por la Iglesia. Los hombres y mujeres mantienen papeles muy tradicionales, los hombres dedicados a los trabajos agrícolas mientras ellas cuidan de los hijos y la familia, las nuevas generaciones muestran empeño y ganas de sacar adelante a su región, accediendo a la educación y preparándose para desarrollar oficios que permitan su progreso.

12 Lamas, Martha, “Cuerpo e identidad”, en: Arango, León y Viveros (compiladoras), Género e identidad. Ensayos sobre lo femenino y lo masculino, Tercer Mundo Editores-Ediciones Uniandes, Bogotá, 1995.

13 Caicedo, C., Gómez, F., Bernal, M., y García, C., Violencia intrafamiliar. Masculinidades y violencia intrafamiliar, Politica Nacional de Construcción de Paz y Convivencia Familiar "Haz Paz", Bogotá: Presidencia de la República de Colombia, 2001.

14 Datos extraídos de la cartilla Catambuco, tierra fértil, 2007. 
Tras realizar la investigación, se puede resaltar la importancia de trabajar estos temas con diferentes poblaciones, siendo una de las principales la de los adolescentes, ya que son ellos quienes están en la búsqueda de su identidad, transcurriendo por el estrecho túnel de los estereotipos ofrecidos por el ambiente que los rodea, entre ellos la escuela, la familia, la iglesia, los medios de comunicación y la sociedad en general, quienes les brindan alternativas de vida. El consolidar su propia identidad es una etapa normal por la que pasa cualquier persona al llegar a la adolescencia, es ahí cuando se da esa búsqueda de su propia esencia: saber quién es, cómo es y qué quiere hacer.

Los modelos juegan un importante papel en este proceso, ya que a través de ellos los adolescentes identificarán hombres y mujeres a los cuales parecerse. Por eso las personas que ellos consideran como ideales son fundamentales, pues muchas veces pueden marcarlos para siempre. Muchos de los modelos de identidad femenina y masculina que nos ofrece el medio social están marcados por elementos ideológicos e incluso comerciales, que promueven ideales para las generaciones emergentes. Cada adolescente tiene ante sí esta tarea primordial. Padres y maestros pueden ayudar ofreciéndole alternativas y ejemplos, pero es cada uno, con base en sus tenencias, inclinaciones, anhelos y experiencias personales y de su grupo de amigos, quien determina cuáles son sus modelos a seguir. Un aspecto a resaltar dentro de la metodología de la investigación fue la conformación del colectivo de adolescentes Jóvenes Construyendo Identidad (JCI), ya que permitió abordar la temática de la identidad de género de una forma que no fuera agresiva con la cultura del corregimiento de Catambuco - caracterizada por la persistencia de estereotipos machistas- ya que esto podría haber sido contraproducente.

Para finalizar es pertinente retomar el título con el que se inició la presente investigación: Identidad de género: ¿Obstáculo al desarrollo o un acceso a la equidad? Tras haber planteado que la cuestión de la identidad de género en los adolescentes se ve mediada por múltiples factores sociales, culturales, familiares y experiencias personales que van consolidando en ellos lo que significa "ser hombre" o "ser mujer", tomando como base los diferentes planteamientos teóricos aportados desde los estudios de género y la interpretación que se realizó por medio de la actividad investigativa, podemos clarificar que en nuestra cultura, pese a los planteamientos y acciones encaminadas a la equidad de género, aun existen muchos estereotipos sexistas que truncan la liberación tanto del hombre como de la mujer y por ende su progreso familiar y social.

Conscientes de la problemática investigada y en concordancia con el objetivo de intervención planteado, es necesario fortalecer las representaciones sociales frente a la identidad género en los jóvenes del corregimiento de Catambuco tras la 
implementación de estrategias que fomenten espacios de equidad, porque no sólo basta con ampliar la esfera del conocimiento de la problemática de la desigualdad de género, sino que es necesario asumir posturas críticas y efectivas que continúen con el proceso de cambio, sin desconocer que es un proceso progresivo ya que cada cultura tiene su ritmo de cambio y evolución. Por ende, entender la identidad de género como un acceso a la equidad implica sensibilización en torno al tema, apropiación de los conocimientos obtenidos mediante los estudios de género, una verdadera conscientización del cambio necesario en el que se planteen como bases de la sociedad el respeto por las diferencias y se re-construyan los espacios públicos y privados con el fin de que exista igualdad de oportunidades. Solo así existirá una sociedad para todos y todas.

\section{Conclusiones}

Para los y las adolescentes del corregimiento de Catambuco que formaron parte del colectivo Jóvenes Construyendo Identidad, el concepto de identidad de género está determinado por la acumulación de experiencias, normas y valores morales que se han ido transmitiendo por medio de las diferentes instituciones sociales, como la familia, la iglesia y la escuela. Estos conceptos son un legado cultural de las generaciones anteriores, padres, abuelos, tíos y demás personas significativas e influyentes en su desarrollo, y a la vez están mediados por las experiencias personales y grupales que se dan en el ambiente específico en el que se desarrollan los sujetos.

Las creencias de los jóvenes de Catambuco acerca del concepto de hombre y mujer son definidas desde las características que cada uno considera que tiene. Por lo general se mencionan cualidades físicas y emocionales, haciendo alusión a que las mujeres son quienes se caracterizan por actuar de una manera más emocional y por tanto son más sensibles a todo lo que sucede en su entorno, a diferencia de los hombres que son considerados como los que utilizan la fuerza física y se caracterizan por tener una baja sensibilidad frente a los acontecimiento, lo cual les ha permitido asumir las diferentes situaciones de una manera fría y poco afectiva.

En cuanto al núcleo familiar existen ciertas normas y reglas con respecto a lo que se les permite y no se les permite hacer a hombres y mujeres. El ambiente familiar de los adolescentes de Catambuco se caracteriza por asignar a las mujeres el cumplir y respetar ciertos horarios restrictivos para salir y/o permanecer en la calle. Ellas también deben realizar los oficios del hogar. Por su lado los hombres pueden salir a la calle sin tantas restricciones y las horas de llegada al hogar son mucho más flexibles. Si ellos quieren ayudar en los oficios del hogar, lo pueden 
hacer, pero si no lo hacen no hay problema alguno y no son juzgados por ello. Se evidencia que existen ciertas diferencias en cuanto a las actividades que realizan los y las adolescentes dentro del hogar.

Las actividades realizadas por personas de cada sexo dentro de los diversos ambientes son conocidos como roles sexuales. Estos son aquellos patrones de comportamiento y atributos personales que han sido definidos por la cultura en la que el individuo se ha desarrollado y son vividos como aquel papel que debe desempeńar cada uno ya sea como hombre o mujer. En nuestra cultura estos roles se han caracterizado por llevar un orden patriarcal que favorece lo masculino sobre lo femenino. Sin embargo, se han dado cambios frente a la concepción que consideraba a ciertas actividades laborales como exclusivas de determinado sexo y han aparecido nuevos roles para ambos géneros dentro de la esfera social y familiar. Los adolescentes manifestaron que tanto hombres como mujeres pueden y son capaces de realizar las mismas actividades en los diferentes cargos laborales; aunque ellos mencionan que hay ciertas actividades que necesitan gran esfuerzo físico y que las mujeres no las pueden realizar por su condición física.

A medida que ha pasado el tiempo se ha evidenciado que aquellas conductas machistas que consideraban la superioridad del hombre sobre la mujer han cambiado, ya que los adolescentes sienten que hoy en día hay una creciente tendencia hacia la consideración de iguales capacidades y condiciones para ambos géneros, en donde las mujeres pueden realizar actividades tanto en el ámbito familiar como ejercer cargos laborales en diferentes áreas y jerarquías, sin ninguna discriminación por su sexo. De igual manera, los hombres pueden dedicarse a su hogar y expresar sus emociones sin sentir que están faltando a su hombría. Sin embargo, persisten todavía algunas formas de machismo que promueven conductas de discriminación sexual. En cuanto a la actitud asumida por los y las adolescentes frente al desempeño de los hombres y mujeres de su región, características de ambos sexos son tomadas como modelos y ejemplos a seguir. Entre ellos se rescatan aspectos tales como la dedicación al trabajo y la responsabilidad en las diversas esferas sociales y familiares. 